\title{
Scientific laws of research funding to support citations and diffusion of knowledge in life science
}

\author{
Melika Mosleh ${ }^{1} \cdot$ Saeed Roshani ${ }^{2} \cdot$ Mario Coccia $^{3}$
}

Received: 10 July 2021 / Accepted: 8 February 2022 / Published online: 5 March 2022

(c) The Author(s) 2022

\begin{abstract}
One of the main problems in scientometrics is to explore the factors that affect the growth of citations in publications to identify best practices of research policy to increase the diffusion of scientific research and knowledge in science and society. The principal purpose of this study is to analyze how research funding affects the citation-based performance of scientific output in vital research fields of life science, which is a critical province (area of knowledge) in science to improve the wellbeing of people. This study uses data from the Scopus database in 2015 (to assess the impact on citations in 2021, after more than 5 years) concerning different disciplines of life science, given by "agricultural and biological sciences", "biochemistry, genetics, and molecular biology", "Immunology and microbiology", "neuroscience" and "pharmacology, toxicology and pharmaceutics". Results demonstrate that although journals publish un-funded articles more than funded publications in all disciplines of life science, the fraction of total citations in funded papers is higher than the share in the total number of publications. In short, funded documents receive more citations than un-funded papers in all research fields of life science under study. Findings also support that citations of total (funded + un-funded), funded, and un-funded published papers have a power-law distribution in all five research fields of life science. Original results here reveal a general property in scientific development: funded research has a higher scaling potential than un-funded publications. Critical implications of research policy, systematized in a decision-making matrix, suggest that R\&D investments in "Neuroscience" can generate a positive impact of scientific results in science and society-in terms of citations-higher than other research fields in medicine. Overall, then, results here can explain some characteristics driving scientific change and help policymakers and scholars to allocate resources towards research fields that facilitate the development and diffusion of scientific research and knowledge in life science for positive societal impact.
\end{abstract}

Keywords Research funding $\cdot$ Research policy $\cdot$ Decision-making matrix $\cdot$ Science diffusion $\cdot$ Scientific development $\cdot$ Power-law distribution

Melika Mosleh

mxm1219@alumni.bham.ac.uk

Extended author information available on the last page of the article 


\section{Introduction}

Several studies in scientometrics have investigated the role of different factors in scientific change, such as public research labs, publications, emerging research fields, nations' research performance, etc. (Coccia, 2005a, 2005b, 2009, 2019a; Coccia et al., 2015; Pagliaro \& Coccia, 2021; Radicchi et al., 2008; Yan et al., 2018; Zhao et al., 2018). A central question in social studies of science is how to enhance the diffusion of scientific research in vital research fields, such as medicine, to increase societal impact. In scientometrics, the scientific performance of papers has been investigated by many scholars, such as Li et al. (2013) that focus on the role of authorship network's impact on publication performance. Scholars also analyze the effects of institutions' collaboration on citation performance, including ranking of academic affiliation (Petersen, 2014) or the scaling relationship between journals' impact and scientific performance (Larivière, 2010; Thelwall \& Wilson, 2014). Other studies investigate the impact of multiple funding on citation performance (MacLean, 1998), the impact factor of journals (Campanario et al., 2011), the relationship between public/private funding and scientific production in nanotechnology (Beaudry, 2012), the role of research sponsorship on research performance in nanoscience (Wang \& Shapira, 2011), etc. Reed et al. (2007) show that the quality of published research is associated with their funding status in medical education. Gök et al. (2015) suggest that citation impact is associated positively with funding diversification and negatively with funding intensity. Checchi (2019) introduces a performance-based research funding system and analyzes its effect on the quantity and quality of different nations' publications. Yan et al. (2018) maintain that funded rather than un-funded publications receive more citations. Quinlan et al. (2008) point out that funded publications attract more citations than un-funded documents. Scholars also argue that funding mainly affects the citations, and funded papers can attract more usage, though a variation exists between research fields (Morillo, 2020; Pao, 1991). Zhao et al. (2018) suggest that research funding is an essential resource in science's reward system. Another study shows that participating in a competition to get the grant regardless of the result can help scientists to accelerate their citations because of their efforts to prepare the proposal, communicate with other authors to get the grant, etc. (Ayoubi et al., 2019). Heyard et al. (2021) analyze the relationship between funding and authors' altered metrics scores and show that funded studies got more public attention than other studies.

Moreover, Fleming et al. (2019) interestingly investigate the impact of funding on innovation according to patents performance and claim that federal supports accelerate innovation production. In general, the literature suggests that funded research has a more substantial impact than un-funded research in science that is magnified by other factors, such as collaboration or authorship affiliation (Rigby, 2013). Financial resources can accelerate the diffusion of science and new knowledge to support society's wellbeing (Laudel, 2006; Roshani et al., 2021). Some studies also suggest no significant relationship between funding and publication performance in some areas (Jacob \& Lefgren, 2011).

In the presence of inconsistencies and ambiguities in the literature on these topics, the relationship between research funding by agencies and the level of papers' citations and diffusion of knowledge in the life science research field is hardly known. In fact, life science currently plays a central role in supporting policy responses and drug discovery processes to cope with the COVID-19 pandemic crisis (Coccia, 2021a, 2021b, 2021c, 2021d, 2021e, 2021f). Stimulated by these fundamental problems and gaps in the literature for a vital scientific domain, this study aims to analyze the relationship between the 
citation-based performance of articles and their funding status in disciplines of life science that play a vital role in improving people's wellbeing. The assumption behind this study is that we just considered the funded publications by agencies in journals which are recorded in global dataset, showing status concerning funding of papers. This study focuses on the investigation of the relationship between funding and publication performance (measured with citations) in life science to find out, as far as possible, general characteristics of the scientific change that can explain science dynamics and support research policies directed to improve the effectiveness of funding allocation for knowledge creation and diffusion in science and society. This study applies an approach of computational scientometrics (the application of bibliometric and scientometrics methods to large-scale datasets) to analyze these topics and suggest implications of research funding policy. The paper here is part of a large body of research that endeavors to successfully identify the driving factors of the evolution of science and technology to support best practices of research and innovation policy in society (cf., Coccia \& Wang, 2016).

\section{Theoretical background}

In order to examine the relationship between funding and citation impact, some studies are reported here to review the current state-of-the-art in these topics. Several studies have investigated different aspects of scientific performance, including the number of citations, publications, word occurrences across documents, collaborations, emerging disciplines, etc. (Peterson, 2010: Coccia et al., 2021). Aksnes et al. (2019) argue that citations are increasingly used as performance indicators within the research system, and scientists with high reputation and many citations will attract more scientific opportunities (e.g., grants, international collaborations, etc.), leading to better scientific performance and further recognition. de Solla-Price (1976) claims that the citation performance of a paper depends on its previous citations. In addition, the highest cited articles appear prior to others in the search engine results, increasing popularity and citations (Aksnes et al., 2019). Scholars show that a scaling relationship can exist between the number of publications and citations (cf., Barabási \& Albert, 1999; Ferraro et al., 2009; Merton, 1988; Tahamtan et al., 2016; Glänzel, 2007). Ronda-Pupo and Katz (2018) show the existence of a scaling relationship among scientific resource performance features. Van Raan (2008) analyzes the scaling relationship between citations and field citation density to demonstrate that top performance universities tend to publish more papers in journals with high impact than lower performance universities. Results also suggest that top performance universities have more opportunities to collaborate with other universities, receive more funds, and get popularity among scholars, encouraging the journals to be more likely to publish their scientific results (Coccia \& Bozeman, 2016; Hicks \& Katz, 2011). Laudel (2006) maintains that researchers rewarded with funding are more likely to receive funds for their future studies. Other studies show that authors who have received grants and a higher level of funding can receive more citations than non-funded papers (Amara et al., 2015). Especially, scientists identify similar patterns in many bibliometric data that lead to the Matthew effect, given by: "the accruing of greater increments of recognition for particular scientific contributions to scientists of considerable repute and the withholding of such recognition from scientists who have not yet made their mark" (Gillespie, 2015; Merton, 1968, 1988; Ye \& Rousseau, 2008). In this regard, Roshani et al. (2021) find that combined, funded, and unfunded research citations follow a power-law distribution in computer science, medicine, 
and economics. Roshani et al. (2021) also show a stronger scaling relationship in funded documents than un-funded papers in the previously mentioned research fields. In short, funded documents received more citations than un-funded papers in computer science, medicine, and economics, and consequently, the Matthew effect is greater for funded articles compared to un-funded documents. Furthermore, papers receiving industry funding can have more citations if their scientific results are pro-industry (Farshad et al., 2013; Kulkarni et al., 2007). Instead, some studies show a low association between citations and grants (Boyack \& Börner 2003).

In order to extend this research stream (Roshani et al., 2021), the study here investigates the relationship between funded and un-funded paper counts and the number of citations in life science, as well as the existence of a power-law that can clarify the scientific change of research fields and help policymakers in making efficient decisions regarding sponsoring specific research fields of life science that can foster the scientific development with fruitful effects for the wellbeing of people in society.

\section{Materials and methods}

\section{Research questions and motivation of the study}

The main research questions of this study are:

- How is the relationship between research funding and citations in life science publications?

- Are there any differences between the growth of the citations in funded and un-funded research in life science?

- Which areas of life science are critical to be funded for major knowledge creation and diffusion?

- Does computational scientometrics provide some decision-making information to support funding in life science?

This study examines these questions to explain, whenever possible, the behavior of citation in funded and un-funded articles of life science and how funding can impact on citations for the diffusion of scientific research and knowledge in science and society.

\section{Sample and sources}

This study focuses on research fields of life science, considering data from the Scopus database (Scopus, 2021). Research fields of life science under study are:

- Agricultural and biological sciences.

- Biochemistry, genetics and molecular biology.

- Immunology and microbiology.

- Neuroscience.

- Pharmacology, toxicology, and pharmaceutics.

These five research fields are the most representative and important life science research areas in the Scopus database classification and method of inquiry here is based on a 
comparative analysis to detect similarity and differences (Coccia \& Benati, 2018; Coccia, 2018a). Data are all publications in 2015 for five research fields of life science just mentioned, using the Scopus database having the number of citation counts (Scopus, 2021). The year 2015 is chosen to have a period of more than five years, until 2021, to assess the performance of publications with their citations. The publication types are limited to articles and reviews published in journals. The string search for retrieving agricultural and biological sciences data is SUBJAREA (AGRI), refined by document types: (Articles OR Review). We changed the SUBJAREA value to "BIOC", "IMMU", "NEUR", and "PHAR" to collect data related to the fields of biochemistry, genetics, and molecular biology, immunology and microbiology, the category of neuroscience, and lastly, pharmacology, toxicology, and pharmaceutics.

\section{Measures}

This study analyzes the papers published in 2015, considering a time lag of more than 5 years for assessing citations adequately over time in "agricultural and biological sciences", "biochemistry, genetics and molecular biology", "immunology and microbiology", "neuroscience", and "pharmacology, toxicology and pharmaceutics".

Funding variables categorize articles and their citations into two categories:

- Funded articles pertain to articles that are published in journals having a funding for performing the scientific research.

- Un-funded articles are the published articles in journals that had not received any funding.

- Total category is based on funded and un-funded articles.

The number of citations is the main indicator of citation-based performance in these different sets mentioned: funded, un-funded, and the Total (funded and un-funded) articles of disciplines under study (Scopus, 2021).

\section{Theoretical and empirical strategy and data analysis procedure}

The analysis of research questions is developed through three main steps.

Firstly, this study focuses on the power-law distribution of Total citations in (funded + un-funded)/funded/un-funded articles published in "agricultural and biological sciences", "biochemistry, genetics and molecular biology", "immunology and microbiology", "neuroscience", and "pharmacology, toxicology and pharmaceutics". The power-law distribution is a suitable model to assess the relationship between the number of publications and their performance with citations according to funding status, as confirmed by other studies (Alstott, et al., 2014; Ronda-Pupo \& Katz, 2016, 2018; Roshani et al., 2021).

Secondly, we measured the power-law correlation between citations and numbers of Total (funded + un-funded)/funded/un-funded publications.

Power-law distribution verification. This study applies the procedure by Clauset et al. (2009, p. 3) to analyze the power-law distribution of the citations of Total (funded+unfunded)/funded/un-funded articles. Xmin value and scaling parameter $\alpha$ of the power-law are calculated. Xmin is the number of publications when the power-law scaling starts 
from this level (Clauset et al., 2009). To measure the Xmin value, we used the formula by Clauset et al. (2009):

$$
\widehat{\alpha} \simeq 1+n\left[\sum_{i=1}^{n} \ln \frac{x_{i}}{x_{\min }-\frac{1}{2}}\right]^{-1}
$$

Throughout this study, we apply the maximum probability method to calculate the parameter $\alpha$. We calculated the fitting goodness of power-law through the data afterwards. We used the bootstrap function of the Monte-Carlo simulation method with a disturbance rate of 2500 to identify whether the power-law distribution can be a plausible pattern for data in life science. Finally, we calculated the Kolmogorov-Smirnov (KS) statistics to measure the fitness of the power-law model for each sample individually. We calculate, in this step, the $p$-value of the model. In power-law distribution, the $p$-value is consistent whenever it is higher than 0.1 , indicating an adequate distribution for the data.

Thirdly, the power-law correlation between the number of citations and the number of (funded+un-funded)/funded/un-funded papers in disciplines of life science is analyzed. Citation-based performance (CBP) relates to all citations obtained by publications. $\mathrm{CBP}$ is a response variable in model, whereas the number of papers published in journals is the explanatory variable. The model by Ronda-Pupo and Katz (2016) is applied to test the power-law correlations between citations and Total (funded+un-funded)/funded/un-funded articles published in life science journals:

$$
C B P=k c^{\alpha}
$$

$C B P=$ Citation-Based Performance, $c=$ number of articles (total: funded+un-funded, funded and un-funded), $k=$ constant, $\alpha=$ scaling factor.

Parameter $\alpha$ is a proxy of the Matthew effect to show the scaling correlation exponent between articles published in journals and their citations. The Ordinary Least Squares (OLS) method estimates the parameter $\alpha$ and the parameter $k$ (Leguendre \& Leguendre, 2012). The scaling exponent $\alpha$ can be used to predict the behavior of correlation. In particular (Ronda-Pupo, 2016):

- Scaling exponent $\alpha>1$, then CBP does not scale linearly, and the number of citations increases at a higher rate than the number of published articles in life science journals. Thus, correlations are nonlinear, and we have a positive cumulative advantage.

- Scaling exponent of $\alpha<1$, then correlation is sub-linear: there is a cumulative disadvantage, which means that the research citations increase slowly compared to the publication rate in journals.

- Scaling exponent of $\alpha=1$ shows a linear correlation between variables, such that the number of citations and journal publications increase at the same rate of growth.

Afterward, we employed a $t$ test to confirm if the scaling exponents of the power-law correlation have statistically a significant level.

Finally, results can be used to suggest a nine-box matrix that is a tool used in research policy to analyze scientific performance in terms of citations of research fields based on funded and/or un-funded publication to support policymakers in R\&D investments of promising research fields. This matrix combines two dimensions: the $x$-axis of diagram represents the alpha value of funded articles categorized (with an assumption of equal 
distribution) in low, medium, and high levels; the $y$-axis represents the alpha value of un-funded publications with the same classification. The relationships between alpha of funded and un-funded papers in funding matrix indicate the strategic positioning of research fields (in boxes or zones) considering the Matthew effect for supporting critical aspects of research funding: Critical, Essential, Irrelevant, Excessive and Futile (Fig. 1).

In this regard, five concepts that can prioritize research funding in different fields are defined considering the alpha of funded articles on the $x$-axis and alpha of un-funded papers on the $y$-axis:

Critical a high gap between alphas of funded and un-funded articles shows that funding might greatly impact citation growth, meaning that financial support policy in this field should be a critical and primary aspect for citation performance improvement.

Essential a medium gap between alphas of funded and un-funded articles; all fields positioned in the essential area should have a secondary priority level in funding policy. Irrelevant funded and un-funded alphas are in the same section (low and low, medium and medium, or high and high). Funding of these research fields does not support citation; accordingly, funding in the research fields included in this section has a lower priority than previous categories.

Excessive the alpha value of funded publications is in a lower section than un-funded research, showing an inverse relationship between funded and citations performance; empirical analysis here shows that funding cannot facilitate citation growth.

Futile funded publications alpha is in the low section, and un-funded alpha is in the high section; in this case, funding might play an inverse role in citation performance and deteriorate the impact of the publication.

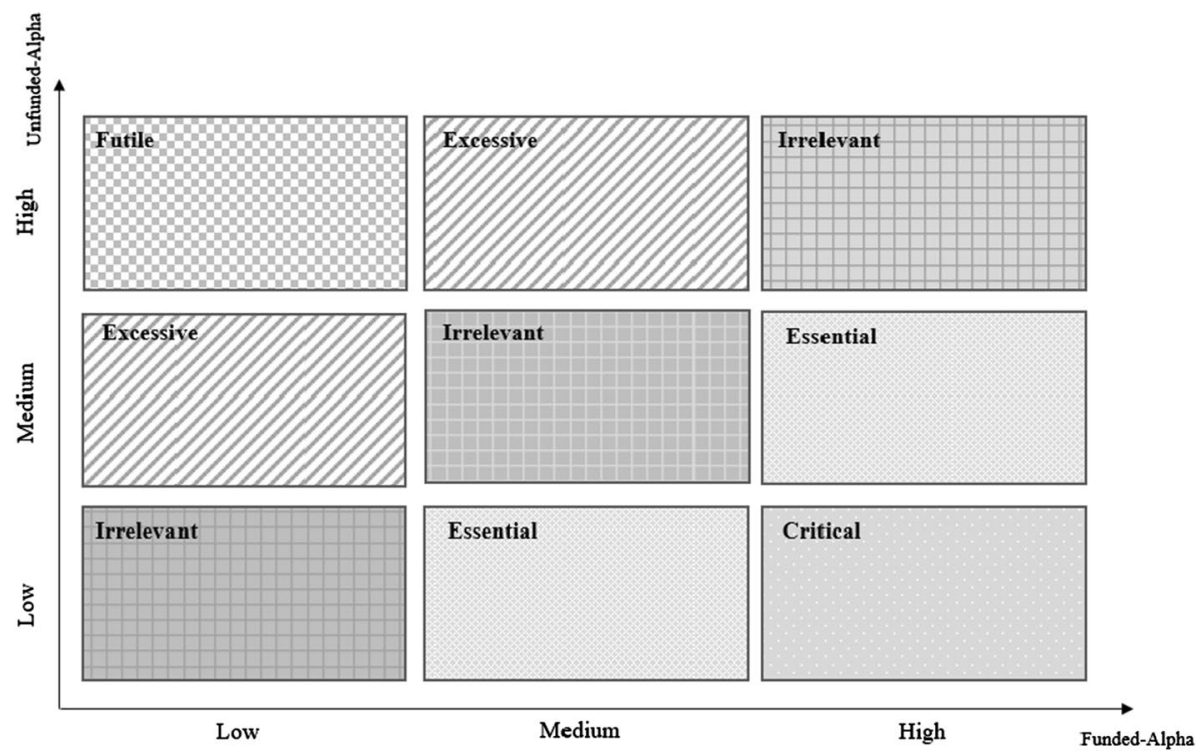

Fig. 1 Theoretical decision-making matrix for decision support of funding research policy in scientific fields 


\section{Results and discussions}

Research fields of life science under study here are:

- Agricultural and biological sciences.

- Biochemistry, genetics and molecular biology.

- Immunology and microbiology.

- Neuroscience.

- Pharmacology, toxicology and phsarmaceutics.

We collected documents from the Scopus (2021) database.

- Accordingly, 187,276 documents are studied in agricultural and biological sciences, including articles and reviews, with 3,044,146 citations published in 1,970 journals. $27.79 \%$ of documents had been supported financially and received $38.67 \%$ of all citations. Moreover, $72.21 \%$ of published papers are un-funded but received a smaller proportion of total citations, around $61.33 \%$.

- We also analyzed 282,182 documents with 6,308,179 citations in 2,006 journals of Biochemistry, Genetics and Molecular Biology. In this field of research, $48.90 \%$ of the citations account for $36.10 \%$ of all documents supported by funding agencies.

- Results also show 65,734 documents in 534 journals of Immunology and Microbiology, in which $46.29 \%$ of citations accounted for $33.24 \%$ of all documents, which are funded papers.

- The neuroscience research field has 62,323 documents in 578 journals, and $56.94 \%$ of its total citations accounted for funded documents (44.86\% of all publications).

- Finally, in 717 Pharmacology, Toxicology and Pharmaceutics journals, results reveal that there are 78,360 records and $43.57 \%$ of citations accounted for financially supported publications (31.40\% of the Total).

Table 1 shows that funded documents in life science journals received more citations than un-funded papers in all five research fields under study, suggesting the beneficial role of sponsorship on the spread of scientific research in this field of research.

Research fields in life science can be categorized in:

- High intensity of research funding, such as biochemistry and neuroscience (number of publications $>35 \%$ ).

- Medium intensity of research funding, including immunology and microbiology, and pharmacology (number of publications: 30-34\%).

- Low intensity of research funding is agricultural and biological sciences (number of publications $<30 \%$ ).

According to Clauset et al. (2009), Xmin is the value when the power-law begins. $\alpha$ is the scaling factor (slope of the log-log regression line). The Kolmogorov-Smirnov (KS) statistic is an indicator to demonstrate the distance between the sample's empirical distribution and the reference's cumulative distribution function.

This study employed Monte-Carlo bootstrapping analysis by 2500 iterations to fit the citations data to a power-law distribution. Table 2 shows the simulation results for five research fields in Total (funded + un-funded), only funded, and only un-funded documents. 
Table 1 The number of documents and citations in research fields of life science according to funding status

\begin{tabular}{|c|c|c|c|c|c|}
\hline Research fields & Sources & Number of papers & $\%$ & Citations & $\%$ \\
\hline \multirow[t]{3}{*}{ Agricultural and biological sciences } & Total $^{\mathrm{a}}$ & 187,276 & 100 & $3,044,146$ & 100 \\
\hline & Funded & 52,048 & 27.79 & $1,177,076$ & 38.67 \\
\hline & Un-funded & 135,228 & 72.21 & $1,867,070$ & 61.33 \\
\hline \multirow[t]{3}{*}{ Biochemistry, genetics and molecular biology } & Total $^{\mathrm{a}}$ & 282,182 & 100 & $6,308,179$ & 100 \\
\hline & Funded & 101,875 & 36.10 & $3,084,843$ & 48.90 \\
\hline & Un-funded & 180,307 & 63.90 & $3,223,336$ & 51.10 \\
\hline \multirow[t]{3}{*}{ Immunology and microbiology } & Total $^{\mathrm{a}}$ & 65,734 & 100 & $1,477,313$ & 100 \\
\hline & Funded & 21,847 & 33.24 & 683,797 & 46.29 \\
\hline & Un-funded & 43,887 & 66.76 & 793,516 & 53.71 \\
\hline \multirow[t]{3}{*}{ Neuroscience } & Total $^{\mathrm{a}}$ & 62,323 & 100 & $1,425,246$ & 100 \\
\hline & Funded & 27,956 & 44.86 & 811,597 & 56.94 \\
\hline & Un-funded & 34,367 & 55.14 & 613,649 & 43.06 \\
\hline \multirow[t]{3}{*}{ Pharmacology, toxicology and pharmaceutics } & Total $^{\mathrm{a}}$ & 78,360 & 100 & $1,257,310$ & 100 \\
\hline & Funded & 24,600 & 31.40 & 547,863 & 43.57 \\
\hline & Un-funded & 53,760 & 68.60 & 709,447 & 56.43 \\
\hline
\end{tabular}

${ }^{\text {a }}$ Total: the combination of funded and un-funded publications

Table 2 Results of fitting the power-law to the datasets

\begin{tabular}{|c|c|c|c|c|}
\hline Research fields & Funding & $X \min$ & $\alpha$ & KS \\
\hline \multirow[t]{3}{*}{ Agricultural and biological sciences } & Total $^{\mathrm{a}}$ & 4.574 & $2.45^{* * *}$ & 0.052 \\
\hline & Funded & 4.009 & $2.68 * * *$ & 0.063 \\
\hline & Un-funded & 2.880 & $2.59 * * *$ & 0.038 \\
\hline \multirow[t]{3}{*}{ Biochemistry, genetics and molecular biology } & Total $^{\mathrm{a}}$ & 5.626 & $2.32 * * *$ & 0.043 \\
\hline & Funded & 6.435 & $2.41 * * *$ & 0.052 \\
\hline & Un-funded & 3.341 & $2.43 * * *$ & 0.020 \\
\hline \multirow[t]{3}{*}{ Immunology and microbiology } & Total $^{\mathrm{a}}$ & 5.655 & $2.51 * * *$ & 0.064 \\
\hline & Funded & 2.128 & $2.17 * * *$ & 0.079 \\
\hline & Un-funded & 1.631 & $2.30 * * *$ & 0.056 \\
\hline \multirow[t]{3}{*}{ Neuroscience } & Total $^{\mathrm{a}}$ & 8.595 & $3.12 * * *$ & 0.093 \\
\hline & Funded & 4.538 & $2.57 * * *$ & 0.071 \\
\hline & Un-funded & 2.939 & $3.05 * * *$ & 0.075 \\
\hline \multirow[t]{3}{*}{ Pharmacology, toxicology and pharmaceutics } & Total $^{\mathrm{a}}$ & 9.117 & $3.31 * * *$ & 0.070 \\
\hline & Funded & 1.974 & $2.29 * * *$ & 0.104 \\
\hline & Un-funded & 2.159 & $2.81 * * *$ & 0.071 \\
\hline
\end{tabular}

${ }^{\mathrm{a}}$ The combination of funded and un-funded publications

**** Denotes significance when Power-Law $p$-value $>0.1$

The statistical analysis supports the model of power-law distribution for all three status of funding in "agricultural and biological sciences", "biochemistry, genetics and molecular biology", "immunology and microbiology", "neuroscience", and "pharmacology, 
toxicology and pharmaceutics". Surprisingly, "pharmacology, toxicology and pharmaceutics" is the only field in which the Xmin parameter of funded papers is lower than unfunded documents; however, the number of un-funded publications, in general, is more. Therefore, financial resources can generate an efficient impact in this field of research. In particular, the funded category needs a lower initial number of citations to start its positive scaling growth. Table 3 shows the comparative analysis between distributions. The $p$-value shows the significance level, and the log-likelihood ratio test (LR) is the main factor in comparing alternative distributions.

Table 4 shows that the power-law correlation between the number of published papers and their citation-based performance in Total (funded+un-funded), funded, and unfunded categories in all research fields under study are statistically significant with a $p$-value $<0.001$. The results reveal that the Matthew effect is greater in funded documents than un-funded ones in all three status sets of five research fields in life science. Agricultural and Biological Sciences show that the number of funded papers grows, scaling the research citation performance. In Biochemistry, Genetics and Molecular, the Matthew effect of funded category is greater than un-funded one, such that funding has a considerable impact on scientific research expansion. Though a positive Matthew effect in un-funded papers in journals of Immunology and Microbiology, the scaling level is lower than other categories under study. In Neuroscience, the Matthew effect is more significant in funded articles than un-funded ones. In fact, we can expect a higher level of citation growth in this research field by increasing the number of funded documents rather than un-funded research papers. In Pharmacology, Toxicology and Pharmaceutics, the Matthew effect of the funded category is more remarkable than other categories. Hence, it can be concluded that a funding strategy can support the research impacts through a strong scaling growth of citation-based performance of papers in life science.

Table 3 Results of comparing the power-law with alternative distributions

\begin{tabular}{|c|c|c|c|c|}
\hline \multirow[t]{2}{*}{ Research fields } & \multirow[t]{2}{*}{ Funding } & \multirow{2}{*}{$\begin{array}{l}\text { Power-law } \\
p\end{array}$} & \multicolumn{2}{|c|}{ Power-law + cut-of } \\
\hline & & & $L R$ & $p$ \\
\hline \multirow[t]{3}{*}{ Agricultural and biological sciences } & Total $^{\mathrm{a}}$ & 0.29 & 0.01 & 0.99 \\
\hline & Funded & 0.60 & 0.001 & 0.99 \\
\hline & Un-funded & 0.85 & 0.11 & 0.79 \\
\hline \multirow[t]{3}{*}{ Biochemistry, genetics and molecular biology } & Total $^{\mathrm{a}}$ & 0.44 & 0.01 & 0.99 \\
\hline & Funded & 0.61 & -1.28 & 0.10 \\
\hline & Un-funded & 0.93 & -0.18 & 0.82 \\
\hline \multirow[t]{3}{*}{ Immunology and Microbiology } & Total $^{\mathrm{a}}$ & 0.47 & -1.22 & 0.11 \\
\hline & Funded & 0.16 & -1.71 & 0.01 \\
\hline & Un-funded & 0.33 & -1.17 & 0.10 \\
\hline \multirow[t]{3}{*}{ Neuroscience } & Total $^{\mathrm{a}}$ & 0.13 & -0.43 & 0.67 \\
\hline & Funded & 0.56 & -0.90 & 0.30 \\
\hline & Un-funded & 0.33 & -1.07 & 0.16 \\
\hline \multirow[t]{3}{*}{ Pharmacology, toxicology and pharmaceutics } & Total $^{\mathrm{a}}$ & 0.50 & -1.03 & 0.16 \\
\hline & Funded & 0.01 & -2.01 & 0.002 \\
\hline & Un-funded & 0.85 & -0.21 & 0.42 \\
\hline
\end{tabular}

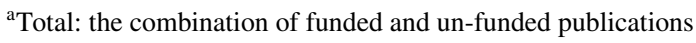


Table 4 Values of the exponents for the power-law correlation

\begin{tabular}{|c|c|c|c|c|c|c|}
\hline Research fields & Funding & $\alpha$ & $\Delta$ & (SD) & $R^{2}$ & $N$ \\
\hline \multirow[t]{3}{*}{ Agricultural and biological sciences } & Total $^{\mathrm{a}}$ & $1.35^{* * *}$ & & $(0.01)$ & 0.74 & 1970 \\
\hline & Funded & $1.30 * * *$ & 0.04 & $(0.01)$ & 0.83 & 1376 \\
\hline & Un-funded & $1.26 * * *$ & & $(0.01)$ & 0.67 & 1960 \\
\hline \multirow[t]{3}{*}{ Biochemistry, genetics and molecular biology } & Total $^{\mathrm{a}}$ & $1.26^{* * * *}$ & & $(0.01)$ & 0.76 & 2006 \\
\hline & Funded & $1.21 * * *$ & 0.04 & $(0.01)$ & 0.81 & 1294 \\
\hline & Un-funded & $1.17 * * *$ & & $(0.01)$ & 0.68 & 1994 \\
\hline \multirow[t]{3}{*}{ Immunology and Microbiology } & Total $^{\mathrm{a}}$ & $1.32 * * *$ & & $(0.02)$ & 0.79 & 534 \\
\hline & Funded & $1.28 * * *$ & 0.08 & $(0.02)$ & 0.85 & 424 \\
\hline & Un-funded & $1.20 * * *$ & & $(0.03)$ & 0.73 & 533 \\
\hline \multirow[t]{3}{*}{ Neuroscience } & Total $^{\mathrm{a}}$ & $1.28 * * *$ & & $(0.03)$ & 0.74 & 578 \\
\hline & Funded & $1.30 * * *$ & 0.16 & $(0.02)$ & 0.88 & 478 \\
\hline & Un-funded & $1.14^{* * *}$ & & $(0.03)$ & 0.63 & 571 \\
\hline \multirow[t]{3}{*}{ Pharmacology, Toxicology and Pharmaceutics } & Total $^{\mathrm{a}}$ & $1.27 * * *$ & & $(0.03)$ & 0.68 & 717 \\
\hline & Funded & $1.28 * * *$ & 0.10 & $(0.02)$ & 0.85 & 515 \\
\hline & Un-funded & $1.18^{* * *}$ & & $(0.03)$ & 0.59 & 707 \\
\hline
\end{tabular}

$p$-value $<0.001$

${ }^{\mathrm{a}}$ Total $=$ funded + un-funded; $\alpha$ is the scaling factor, $\Delta=$ Difference between $\alpha$ (funded)- $\alpha$ (un-funded), SD Standard Deviation, $R^{2}$ is the coefficient of determination, $N$ Number of cases

Table 4 also shows that coefficients of $R^{2}$ of the funded articles in all five disciplines of life science are higher than $R^{2}$ of the power-law model for un-funded papers, suggesting a better goodness of fit.

To conduct a visual comparison of the Matthew effect based on the power-law correlation between the group of funded and un-funded publications, we classify the results of scaling exponent in three categories according to the intensity of the Matthew effect (Table 5):

- High Matthew effect has $\alpha \geq 1.29$.

- Medium Matthew effect has $1.18<\alpha<1.29$.

- Low Mathew effect has $\alpha \leq 1.18$ ).

Remark The thresholds are calculated using the 25th and 75th percentiles of the distribution of estimated $\alpha$ for power-law model (including funded and un-funded data): 25 th percentiles (lower) is 1.18; the 75th percentiles (higher) is 1.29 .

These results show that funded publications in "Agricultural and Biological Sciences" and "Neuroscience" have a higher Matthew effect compared to other categories, and a funding research policy in such disciplines leads to a higher rate of scaling in citation growth (Table 5). Thus, a research policy implication is that funding these scientific fields of life science can positively impact on scientific performance and knowledge diffusion in society. In general, citations of funded publications in all five disciplines at 


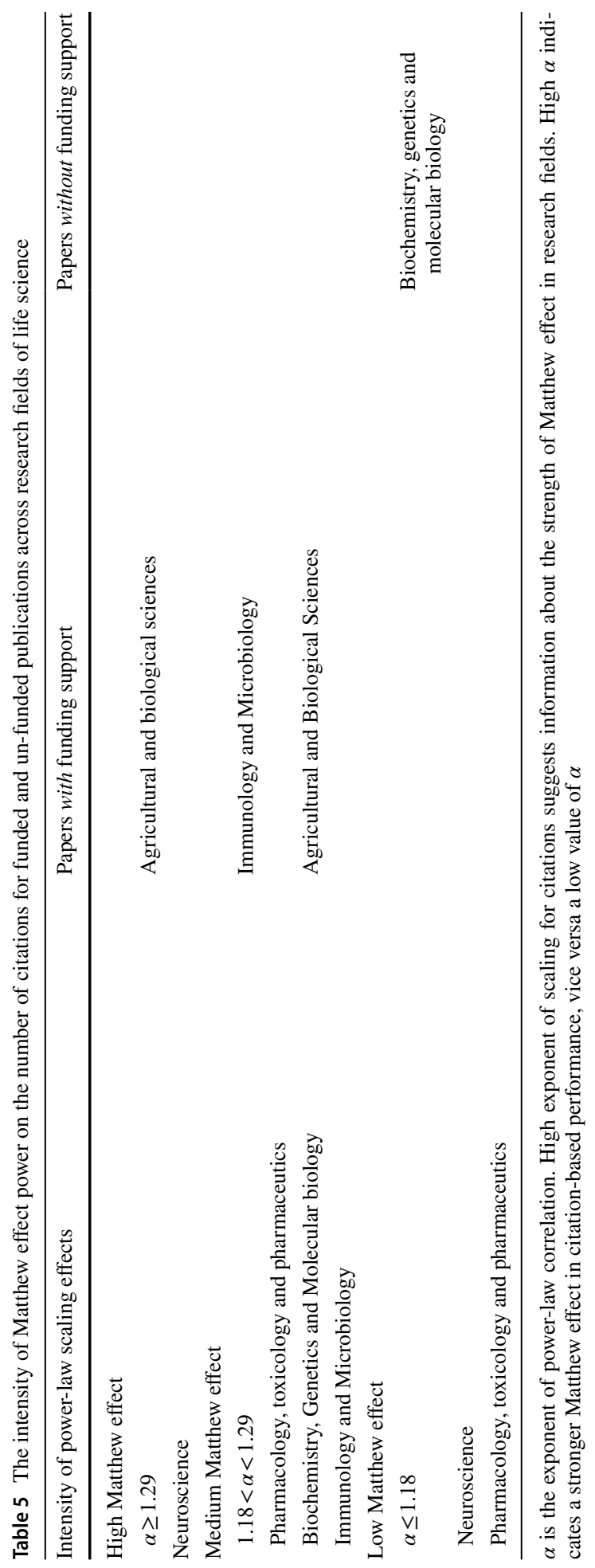


least scale exponentially at a medium level. Moreover, results suggest that citations of un-funded publications have a medium and lower intensity of Matthew effect; none of them has experienced a high level of Matthew effect in their citation-based performance growth.

Table 6 suggests three levels of Matthew effect between funded and un-funded papers to interpret better the results and recommend more efficient research policy implications considering funding strategies.

According to the results here, to conduct an effective policy of research funding to increase the scientific performance of citations of papers in life science, "Neuroscience" with high Matthew effect with funding and high Matthew effect gap $(\Delta=\alpha$ funded- $\alpha$ un-funded) should position in a high priority area for R\&D investments in life science. It means that citations of funded publications grow with a high scaling exponent by increasing the number of publications, and this scaling effect is significantly greater than scaling effect of un-funded publications. In "Pharmacology, Toxicology and Pharmaceutics", since delta $(\Delta)$ is at a medium level, investing in this field can also be considered an effective research policy. "Immunology and Microbiology" have both a medium level of Matthew effect gap and Matthew effect power in funded category. Although there is a powerful Matthew effect in funded categories of "Agricultural and Biological Sciences", citations of both un-funded and funded publications tend to increase regardless of their funding status. Indeed, this deficiency in the performance of funding strategy is greater in "Biochemistry, Genetics and Molecular Biology": since there is no considerable gap between the scaling exponent of funded and un-funded sets of publications, this research field has a low priority in R\&D investment to support citation growth and science diffusion in life science compared to other fields.

Finally, the results of Tables 5 and 6 here can be used to suggest a logical scheme represented in Fig. 2 directed to support policymakers in R\&D investments. In Fig. 2, the $x$-axis represents the alpha value of funded articles, categorized in Low $(\alpha \leq 1.18)$, Medium $(1.18<\alpha<1.29)$, and High $(\alpha \geq 1.29)$. The $y$-axis shows the alpha value of un-funded publications with the same categorization: Low, Medium, High. The relationships between alpha of funded and un-funded papers in the scheme of Fig. 2, as explained in methods, are a useful criterion to support funding of research fields considering the strength of Matthew effect in both funded and un-funded publications, as follows:

Critical zone: A high gap between alphas of funded and un-funded articles shows that funding greatly impacts citation growth, and that financial support policy in this field should be a critical and primary aspect for improvement of citation performance, such as in neuroscience

Essential zone: High funded Matthew effect and medium un-funded Matthew Effect, or medium funded Matthew effect and low un-funded Matthew effect; funding research policy here can boost the citation impact exponentially; moreover, the lower gap between the magnitude of scaling in funded and un-funded categories leads to consider disciplines in this area having a secondary priority of research funding (e.g., Agricultural and Biological Sciences, Biochemistry, Genetics and Molecular Biology; Pharmacology, Toxicology and Pharmaceutics)

Irrelevant zone: Both funded and un-funded categories are in the same level of Low, Medium, or High; it means that both un-funded and funded publications citation will scale exponentially in favor of the increasing number of publications. Although the magnitude of Matthew effect significantly leads to higher citation performance in High-High and Medium-Medium areas, the gap between funded and un-funded categories is insufficient to support a primary funding research policy for these research fields. Additional factors are necessary to support effective decision-making procedures of R\&D funding and investments of research field here-for instance, Immunology and Microbiology 


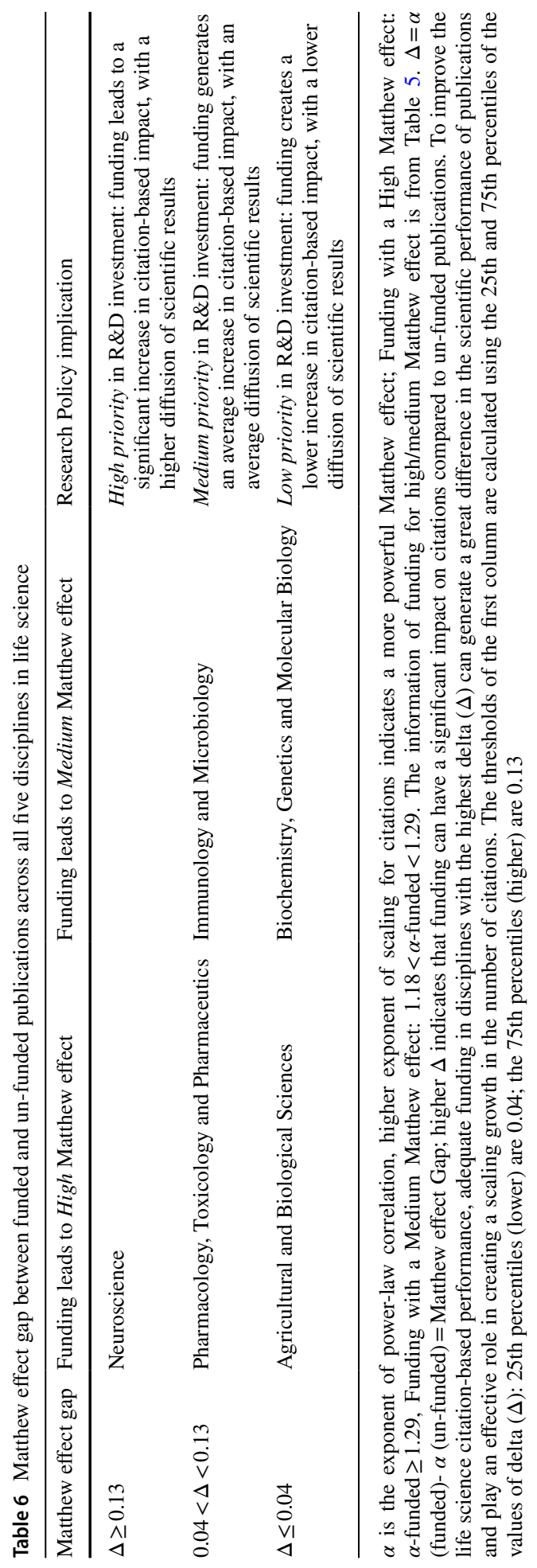




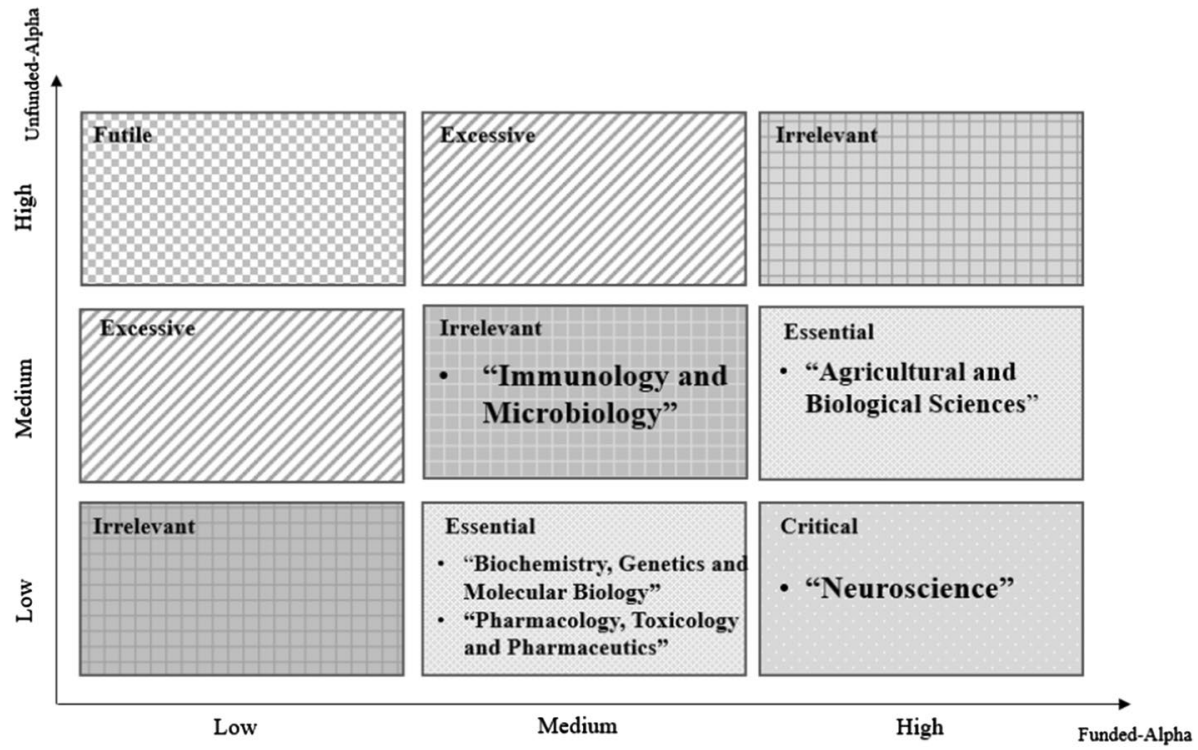

Fig. 2 Decision-making matrix for funding policy of research fields in life science according to their location

Excessive zone: funding in this area does not positively impact on citation-based performance, and R\&D investment policy for these disciplines may be decreased to have more efficient budgeting

Futile zone: funding research policy to support citation performances is unnecessary for research fields in this area. Hence, funding is not an effective strategy in these disciplines to improve scientific performance regarding citations and diffusion of science in society

Figure 2 suggests that a funding policy in "Neuroscience" is critical strategy to improving the scientific impact in life science. Therefore, this field should be supported financially because investing in Neuroscience can greatly improve scientific performance and consequential knowledge diffusion in life science. Furthermore, "Agricultural and Biological Sciences", "Pharmacology, Toxicology and Pharmaceutics", and "Biochemistry, Genetics and Molecular Biology" are fields in which funding research policy is an essential strategy for improving the scientific performance of papers and diffusion of scientific research in society. Finally, funding in "Immunology and Microbiology" seems to be an irrelevant research policy that cannot significantly play a role of accelerator for improving citations of papers; here, it is important to consider other relevant factors to make decisions for R\&D investments. In short, Fig. 2 can support a research policy of funding in different fields to efficiently increase the citation-based performance of publications in life science for widespread scientific research in science and society.

Finally, these findings, based on case study of life science, can have main theoretical implications to clarify aspects of scientific change and support the research policy of scientific fields, given by following properties (or laws): 
1st Law. Number of citations of funded and un-funded publications grows through a power-law distribution

2nd Law. Funded research has a higher scaling potential than un-funded publications 3rd Law. Funded documents receive more citations than un-funded papers in all research fields

4th Law. Funded papers have a more substantial cumulative advantage of citations than un-funded papers

Remark Funding the research field of Neuroscience increases the citation impact of its publications compared with other fields, whereas funding the research field of Immunology and Microbiology does not significantly affect the citation-based performance of published papers in journals.

\section{Concluding observations and limitations}

This study explores the relationship between research funding and the citation-based performance of scientific output in research fields of life science. The methods focus on scientific output of published papers because: (a) scientific output is recorded in on-line databases that are easily accessible, and $(b)$ funding status of papers can be detected and in general is provided by agencies after an external revision and assessment that should be and indicator of quality and support a scientific research and output having a high potential impact in science and society (and consequently a high scientific performance). In this context, the study here analyzes the publications recorded in citation database by Scopus (2021) concerning critical disciplines of life science, given by "agricultural and biological sciences", "biochemistry, genetics and molecular biology", "Immunology and microbiology", "neuroscience" and "pharmacology, toxicology and pharmaceutics". This study does not consider scientific research not published because it is not recorded in datasets, and it is difficult to detect and investigate its funding status due to heterogeneity of content not including all information needed for reliable comparative analyses, such that a scientometric analysis including this research not published may lead to misleading results. In addition, many papers and studies can be funded indirectly by the universities, such as by salary and other benefits that professors and scholars receive from universities and that are not specific resources for doing research, but scholars produce scientific papers for curiosity and other natural characteristics of researchers. Hence, indirect fundings in scientific research are also main aspects but difficult to detect and estimate and including this variable factor can create possible distortions in comparative analyses for assessing the scientific impact, in terms of citation performance, of papers in science and society.

Results here show that although journals publish un-funded articles more than funded publications in life science, funded documents received more citations than un-funded papers. Findings also support that Total (funded+un-funded), funded, and un-funded papers have citations following a power-law distribution in all research fields of life science under study. Our findings confirm previous studies by showing that funding of agencies is a driver to scale the scientific performance of publications, especially citation impact in different disciplines, such as economics, computer science, etc. (Roshani et al., 2021). This main result has been extended here in life science to improve allocation of resources and consequently wellbeing of people and to cope with pandemic crises, such as Coronavirus 
Disease 2019 (COVID-19), that create socioeconomic problems in society (Coccia, 2021a, 2021b, 2021c, 2021d, 2021e, 2021f). This study is connected to many studies of scientometrics in these topics as described in the section of introduction and theoretical framework (Checchi, 2019; Gök et al., 2015; Reed et al., 2007; Zhao et al., 2018). In fact, some studies investigate the relationship between funding and citation impact with different methodologies showing similar results to analyses discussed here. Yan et al. (2018) analyze the relationship between scientific financing and citation impact in science, technology, engineering, mathematics, and medicine (STEMM) area using a regression model with Heckman bias adjustment and show that financing has a significant relationship with a paper's citations in STEMM fields. According to another study by Wagner and Jonkers (2017), public R\&D investment is only tangentially correlated with the citation impact of a nation's publications as evaluated by the field-weighted citation index. Leydesdorff et al. (2019) and Benavente et al. (2012) also point out modest coefficients between public funding and citation impact, using a negative binomial regression analysis. Our study here, by employing a different methodology, shows not only a positive relationship between funding and citations of papers in life science but also a cumulative effect of citations over time. Moreover, in this context, a preliminary analysis (to be further developed in future) considers individual data of leading scholars (awarded with Nobel Prize) to assess how funding can affect citations of their papers. In particular, we consider publications and citations of scholars having Nobel Prize in Chemistry and Medicine over 2019-2020 period (Table 7). Results suggest that in Chemistry funded papers have a higher average citation than unfunded papers, vice versa in Medicine.

These preliminary results suggest that manifold factors, in addition to institutional funding, can affect the citation and diffusion of knowledge in scientific fields, such as reputation of scholars, nature of research fields, researcher characteristics, institutions and countries in which research is developed, life cycle effects of scholars and papers, etc. In general, the effects of funding on citations of papers provide tentative results, and this study is a starting point for further investigation considering not only data at macro level of research fields, but also data at micro level of leading scholars to analyze the complex factors and dynamics between funding and citations to foster the diffusion of knowledge in science and society.

Although this study has provided some interesting and preliminary results, it has several limitations. First, a limitation of this study is that sources understudy may only capture certain aspects of the ongoing dynamics of citations between research fields in life science. Second, multiple confounding factors could have an essential role in the patterns of citation performance in life science at level of research fields to be further investigated (e.g., institutional aspects, investments, network of collaboration, openness, intellectual property rights, etc.). Third, this study's computational and statistical analyses focus on data in a specific period and research fields of life science and studies that found the opportunity to be published in journals. Numerous funded and un-funded papers have not been published

Table 7 Average citations of publications of scholars awarded with Nobel Prize in Chemistry and Medicine in 2019 and 2020

\begin{tabular}{llllll}
\hline 2019-2020 & \multicolumn{2}{l}{ Chemistry } & & \multicolumn{2}{l}{ Medicine } \\
\cline { 2 - 3 } \cline { 5 - 6 } \cline { 5 - 6 } & Funded & Un-funded & & Funded & Un-funded \\
\hline Papers (number) & 530 & 287 & & 1044 & 641 \\
Citations & 95,829 & 31,063 & & 201,583 & 144,305 \\
Average citations & 180.81 & 108.23 & & 193.09 & 225.12 \\
\hline
\end{tabular}


in journals and deserve to be investigated in future studies with appropriate methods that detect this latent area in science. Finally, future studies can also focus, as said, on individual data of scholars for a benchmark analysis to support current results and clarify aspects and changes of the relation under study at micro and macro level of analysis. Thus, generalizing the results of this research should be done with caution 09 .

To conclude, future research should consider more data, and when possible, with new approaches to reinforce proposed findings here (cf., Rodríguez-Navarro \& Brito, 2018, 2019). Despite these limitations, the results here clearly illustrate the vital role of funding for a higher scientific impact and diffusion of knowledge in life science but also the need for more detailed examinations of the relationship between patterns of citations and research funding for appropriate research and innovation strategies to foster scientific development to improve the wellbeing of people in society.

Open Access This article is licensed under a Creative Commons Attribution 4.0 International License, which permits use, sharing, adaptation, distribution and reproduction in any medium or format, as long as you give appropriate credit to the original author(s) and the source, provide a link to the Creative Commons licence, and indicate if changes were made. The images or other third party material in this article are included in the article's Creative Commons licence, unless indicated otherwise in a credit line to the material. If material is not included in the article's Creative Commons licence and your intended use is not permitted by statutory regulation or exceeds the permitted use, you will need to obtain permission directly from the copyright holder. To view a copy of this licence, visit http://creativecommons.org/licenses/by/4.0/.

\section{References}

Aksnes, D. W., Langfeldt, L., \& Wouters, P. (2019). Citations citation indicators and research quality: An overview of basic concepts and theories. SAGE Open, 9(1), 2019. https://doi.org/10.1177/2158244019 829575

Alstott, J., Bullmore, E., \& Plenz, D. (2014). Power law: A python package for analysis of heavy-tailed distributions. PLoS ONE, 9(1), e85777. https://doi.org/10.1371/journal.pone.0085777

Amara, N., Landry, R., \& Halilem, N. (2015). What can university administrators do to increase the publication and citation scores of their faculty members? Scientometrics, 103, 489-530.

Ayoubi, C., Pezzoni, M., \& Visentin, F. (2019). The important thing is not to win, it is to take part: What if scientists benefit from participating in research grant competitions? Research Policy, 48(1), 84-97.

Barabási, A. L., \& Albert, R. (1999). Emergence of scaling in random networks. Science, 286(5439), 509-512.

Beaudry, C., \& Allaoui, S. (2012). Impact of public and private research funding on scientific production: The case of nanotechnology. Research Policy, 41(9), 1589-1606.

Benavente, J. M., Crespi, G., Garone, L. F., \& Maffioli, A. (2012). The impact of national research funds: A regression discontinuity approach to the Chilean FONDECYT. Research Policy 41(8), 1461-1475.

Boyack, K. W., \& Börner, K. (2003). Indicator-assisted evaluation and funding of research: visualizing the influence of grants on the number and citation counts of research papers. Journal of the American Society for Information Science and Technology, 54(5), 447-461.

Campanario, J. M., Carretero, J., Marangon, V., Molina, A., \& Ros, G. (2011). Effect on the journal impact factor of the number and document type of citing records: A wide-scale study. Scientometrics, 87(1), 75-84.

Checchi, D., Malgarini, M., \& Sarlo, S. (2019). Do performance-based research funding systems affect research production and impact? Higher Education Quarterly, 73(1), 45-69.

Clauset, A., Shalizi, C. R., \& Newman, M. E. J. (2009). Power-law distributions in empirical data. SIAM Review, 51(4), 661-703.

Coccia, M. (2005a). A taxonomy of public research bodies: A systemic approach. Prometheus, 23(1), 63-82. https://doi.org/10.1080/0810902042000331322

Coccia, M. (2005b). A Scientometric model for the assessment of scientific research performance within public institutes. Scientometrics, 65(3), 307-321. https://doi.org/10.1007/s11192-005-0276-1 
Coccia, M. (2009). Research performance and bureaucracy within public research labs. Scientometrics, 79(1), 93-107. https://doi.org/10.1007/s11192-009-0406-2

Coccia, M. (2015). Spatial relation between geo-climate zones and technological outputs to explain the evolution of technology. International Journal of Transitions and Innovation Systems, 4(1-2), 5-21. https://doi.org/10.1504/IJTIS.2015.074642

Coccia, M. (2018a). General properties of the evolution of research fields: A scientometric study of human microbiome, evolutionary robotics and astrobiology. Scientometrics, 117(2), 1265-1283. https://doi. org/10.1007/s11192-018-2902-8

Coccia, M. (2018b). An introduction to the methods of inquiry in social sciences. Journal of Social and Administrative Sciences, 5(2), 116-126. https://doi.org/10.1453/jsas.v5i2.1651

Coccia, M. (2019a). Why do nations produce science advances and new technology. Technology in Society, 59(101124), 1-9. https://doi.org/10.1016/j.techsoc.2019.03.007

Coccia, M. (2019b). Metabolism of public research organizations: How do laboratories consume state subsidies? Public Organization Review.

Coccia, M. (2020b). How (Un)sustainable environments are related to the Diffusion of COVID-19: The relation between coronavirus disease 2019 air pollution wind resource and energy. Sustainability, 12(22), 9709. https://doi.org/10.3390/su12229709

Coccia, M. (2020a). The evolution of scientific disciplines in applied sciences: Dynamics and empirical properties of experimental physics. Scientometrics, 124, 451-487. https://doi.org/10.1007/ s11192-020-03464-y

Coccia, M. (2021e). The relation between length of lockdown, numbers of infected people and deaths of COVID-19 and economic growth of countries: Lessons learned to cope with future pandemics similar to COVID-19. Science of the Total Environment, 775, 145801. https://doi.org/10.1016/j.scitotenv. 2021e. 145801

Coccia, M. (2021a). High health expenditures and low exposure of population to air pollution as critical factors that can reduce fatality rate in COVID-19 pandemic crisis: A global analysis. Environmental Research, 199, 111339. https://doi.org/10.1016/j.envres.2021a.111339

Coccia, M. (2021f). Evolution of technology in replacement of heart valves: Transcatheter aortic valves, a revolution for management of valvular heart diseases. Health Policy and Technology, 10(2), 100512. https://doi.org/10.1016/j.hlpt.2021f.100512

Coccia, M. (2021c). Evolution and structure of research fields driven by crises and environmental threats: The COVID-19 research. Scientometrics. https://doi.org/10.1007/s11192-021-04172-X

Coccia, M. (2021d). Effects of the spread of COVID-19 on public health of polluted cities: Results of the first wave for explaining the dejà vu in the second wave of COVID-19 pandemic and epidemics of future vital agents. Environmental Science and Pollution Research., 28(15), 19147-19154. https://doi. org/10.1007/s11356-020-11662-7

Coccia, M. (2022). Probability of discoveries between research fields to explain scientific and technological change. Technology in Society, 68, 101874. https://doi.org/10.1016/j.techsoc.2022.101874

Coccia, M., \& Benati, I. (2018). Comparative Models of Inquiry. In A. Farazmand (Ed.), Global encyclopedia of public administration, public policy, and governance. Heidelberg: Springer International Publishing.

Coccia, M., \& Bozeman, B. (2016). Allometric models to measure and analyze the evolution of international research collaboration. Scientometrics, 108(3), 1065-1084. https://doi.org/10.1007/s11192-016-2027-X

Coccia, M., Falavigna, G., \& Manello, A. (2015). The impact of hybrid public and market-oriented financing mechanisms on scientific portfolio and performances of public research labs: A scientometric analysis. Scientometrics, 102(1), 151-168. https://doi.org/10.1007/s11192-014-1427-z

Coccia, M., Roshani, S., \& Mosleh, M. (2021). Scientific developments and new technological trajectories in sensor research. Sensors, 21(23), 7803. https://doi.org/10.3390/s21237803

Coccia, M., \& Wang, L. (2016). Evolution and convergence of the patterns of international scientific collaboration. Proceedings of the National Academy of Sciences of the United States of America, 113(8), 2057-2061. https://doi.org/10.1073/pnas.1510820113

Coccia, M. (2021a). Pandemic Prevention: Lessons from COVID-19. Encyclopedia 2021b, 1, pp. 433444. MDPI, Basel, Switzerland, Encyclopedia of COVID-19 ISSN 2673-8392, open access journal, (https://www.mdpi.com/journal/encyclopedia) https://doi.org/10.3390/encyclopedia1020036

de Solla Price, D. J. (1976). A general theory of bibliometric and other cumulative advantage processes. Journal of the American Society for Information Science, 27, 292-306.

Farshad, M., Sidler, C., \& Gerber, C. (2013). Association of scientific and nonscientific factors to citation rates of articles of renowned orthopedic journals. European Orthopaedics and Traumatology, 4(3), 125-130. 
Ferraro, K. F., Shippee, T. P., \& Schafer, M. H. (2009). Cumulative inequality theory for research on aging and the life course. In V. L. Bengston, D. Gans, N. M. Pulney, \& M. Silverstein (Eds.), Handbook of theories of aging (pp. 413-433). Springer.

Fleming, L., Greene, H., Li, G., Marx, M., \& Yao, D. (2019). Government-funded research increasingly fuels innovation. Science, 364(6446), 1139-1141.

Fortunato, S., Bergstrom, C. T., Börner, K., Evans, J. A., Helbing, D., Milojević, S., Petersen, A. M., Radicchi, F., Sinatra, R., Uzzi, B., Vespignani, A., Waltman, L., Wang, D., \& Barabási, A. L. (2018). Science of science. Science, 359, 6379. https://doi.org/10.1126/science.aao0185

Gillespie, C. S. (2015). Fitting heavy tailed distributions: The poweRlawpackage. Journal of Statistical Software, 64, 1-16.

Glänzel, W. (2007). Characteristic scores and scales. A bibliometric analysis of subject characteristics based on long-term citation observation. Journal of Informetrics, 1, 92-102.

Gök, A., Waterworth, A., \& Shapira, P. (2015). Use of web mining in studying innovation. Scientometrics, 102(1), 653-671. https://doi.org/10.1007/s11192-014-1434-0

Heyard, R., \& Hottenrott, H. (2021). The value of research funding for knowledge creation and dissemination: A study of SNSF research grants. Humanities and Social Sciences Communications, 8(1), 1-16. https://doi.org/10.1057/s41599-021-00891-X

Hicks, D., \& Katz, J. S. (2011). Equity and excellence in research funding. Minerva, 49(2), 137-151.

Jacob, B. A., \& Lefgren, L. (2011). The impact of research grant funding on scientific productivity. Journal of Public Economics, 95(9-10), 1168-1177. https://doi.org/10.1016/j.jpubeco.2011.05.005

Kashani, E. S., \& Roshani, S. (2019). Evolution of innovation system literature: Intellectual bases and emerging trends. Technological Forecasting and Social Change, 146, 68-80.

Kulkarni, A. V., Busse, J. W., \& Shams, I. (2007). Characteristics associated with citation rate of the medical literature. PLOS ONE, 2(5), e403.

Larivière, V., \& Gingras, Y. (2010). The impact factor's Matthew Effect: A natural experiment in bibliometrics. Journal of the American Society for Information Science and Technology, 61(2), 424-427.

Laudel, G. (2006). The art of getting funded: How scientists adapt to their funding conditions. Science and Public Policy, 33(7), 489-504.

Leguendre, P., \& Leguendre, L. (2012). Numerical ecology (3rd ed.). Elsevier B. V.

Leydesdorff, L., Bornmann, L., \& Wagner, C. S. (2019). The relative influences of government funding and international collaboration on citation impact. Journal of the Association for Information Science and Technology, 70(2), 198-201.

Li, E. Y., Liao, C. H., \& Yen, H. R. (2013). Co-authorship networks and research impact: A social capital perspective. Research Policy, 42(9), 1515-1530.

MacLean, M., Davies, C., Lewison, G., \& Anderson, J. (1998). Evaluating the research activity and impact of funding agencies. Research Evaluation, 7(1), 7-16.

Mario, C. (2019). Metabolism of public research organizations: How do laboratories consume state subsidies? Public Organization Review, 19(4), 473-491.

Merton, R. (1968). The matthew effect in science. Science, 159(3810), 56-63.

Merton, R. K. (1988). The matthew effect in science, II: Cumulative advantage and the symbolism of intellectual property. Isis, 79(4), 606-623.

Morillo, F. (2020). Is open access publication useful for all research fields? Presence of funding, collaboration and impact. Scientometrics, 125, 689-716. https://doi.org/10.1007/s11192-020-03652-w

Pagliaro, M., \& Coccia, M. (2021). How self-determination of scholars outclasses shrinking public research lab budgets, supporting scientific production: A case study and R\&D management implications. Heliyon, 7(1), e05998. https://doi.org/10.1016/j.heliyon.2021.e05998

Pao, M. L. (1991). On the relationship of funding and research publications. Scientometrics, 20, $257-281$. https://doi.org/10.1007/BF02018158

Petersen, A. M., \& Penner, O. (2014). Inequality and cumulative advantage in science careers: A case study of high-impact journals. EPJ Data Science, 3(1), 24.

Peterson, G. J., Pressé, S., \& Dill, K. A. (2010). Nonuniversal power law scaling in the probability distribution of scientific citations. Proceedings of the National Academy of Sciences, 107(37), 16023-16027.

Quinlan, K. M., Kane, M., \& Trochim, W. M. K. (2008). Evaluation of large research initiatives: Outcomes challenges and methodological considerations. New Directions for Evaluation, 118, 61-72.

Radicchi, F., Fortunato, S., \& Castellano, C. (2008). Universality of citation distributions: Toward an objective measure of scientific impact. Proceedings of the National Academy of Sciences of the United States of America, 105, 17268-17272.

Reed, D. A., Cook, D. A., Beckman, T. J., Levine, R. B., Kern, D. E., \& Wright, S. M. (2007). Association between funding and quality of published medical education research. JAMA, 298(9), 1002-1009. 
Rigby, J. (2013). Looking for the impact of peer review: Does count of funding acknowledgements really predict research impact? Scientometrics, 94(1), 57-73.

Rodríguez-Navarro, A., \& Brito, R. (2018). Double rank analysis for research assessment. Journal of Informetrics, 12, 31-41.

Rodríguez-Navarro, A., \& Brito, R. (2019). Probability and expected frequency of breakthroughs: Basis and use of a robust method of research assessment. Scientometrics, 119, 213-235. https://doi.org/10.1007/ s11192-019-03022-1

Ronda-Pupo, G. A., \& Katz, J. S. (2016). The scaling relationship between citation-based performance and coauthorship patterns in natural sciences. Journal of the Association for Information Science and Technology, 68(5), 1257-1265.

Ronda-Pupo, G. A., \& Katz, J. S. (2018). The power law relationship between citation impact and multiauthorship patterns in articles in information science \& library science journals. Scientometrics, 114(3), 919-932.

Roshani, S., Bagherylooieh, M. R., Mosleh, M., \& Coccia, M. (2021). What is the relationship between research funding and citation-based performance? A comparative analysis between critical disciplines. Scientometrics, 126(9), 7859-7874.

Scopus. (2021). Scopu. Searchin:Startexploring,https://www.scopus.com/search/form.uri?display=basic\# basic

Tahamtan, I., Safipour Afshar, A., \& Ahamdzadeh, K. (2016). Factors affecting number of citations: A comprehensive review of the literature. Scientometrics, 107, 1195-1225. https://doi.org/10.1007/ s11192-016-1889-2

Thelwall, M., \& Wilson, P. (2014). Regression for citation data: An evaluation of different methods. Journal of Informetrics, 8, 963-971.

Van Raan, A. F. V. (2008). Bibliometric statistical properties of the 100 largest European research universities: Prevalent scaling rules in the science system. Journal of the American Society for Information Science and Technology, 59(3), 461-475.

Wagner, C. S., \& Jonkers, K. (2017). Open countries have strong science. Nature News, 550(7674), 32.

Wang, J., \& Shapira, P. (2011). Funding acknowledgement analysis: An enhanced tool to investigate research sponsorship impacts: The case of nanotechnology. Scientometrics, 87(3), 563-586.

Yan, E., Wu, C., \& Song, M. (2018). The funding factor: A cross-disciplinary examination of the association between research funding and citation impact. Scientometrics, 115(1), 369-384.

Ye, F. Y., \& Rousseau, R. (2008). The power law model and total career h-index sequences. Journal of Informetrics, 2(4), 288-297. https://doi.org/10.1016/j.joi.2008.09.002

Zhao, S. X., Lou, W., Tan, A. M., \& Yu, S. (2018). Do funded papers attract more usage? Scientometrics, $115(1), 153-168$.

\section{Authors and Affiliations}

\section{Melika Mosleh ${ }^{1} \cdot$ Saeed Roshani ${ }^{2} \cdot$ Mario Coccia $^{3}$}

Saeed Roshani

Roshani@atu.ac.ir

Mario Coccia

mario.coccia@cnr.it

1 Birmingham Business School, College of Social Sciences, University of Birmingham, Birmingham, UK

2 Department of Technology and Entrepreneurship Management, Faculty of Management and Accounting, Allameh Tabataba'i University, Tehran, Iran

3 National Research Council of Italy, Collegio Carlo Alberto, Via Real Collegio, Torino, Italy 